\title{
Manejo de lesión traqueoesofágica completa con anastomosis esofágica y traqueal terminoterminal más esofagostomía con colocación de sonda en T
}

\section{Management of complete tracheo-esophageal injury with esophageal and tracheal end-to-end anastomosis plus esophagostomy with T-tube placement}

\author{
Armando I. Reyes-Carrillo ${ }^{1}$, Enrique Villela-Cañas ${ }^{1}$, José G. Padilla-López ${ }^{1}$, \\ Luis B. Enríquez-Sánchez ${ }^{1 *}$, Marco J. Carrillo-Gorena ${ }^{2}$, Manuel A. Carbajal-Vega', \\ Manuel D. Pérez-Ruiz' ${ }^{1}$ Armando Soto-Pérez², David Hernández-Ríos², César Aragón-Quintana ${ }^{2}$ y \\ David A. Aguirre-Baca ${ }^{3}$
}

${ }^{1}$ Departamento de Cirugía General, Hospital Central del Estado; ${ }^{2}$ División quirúrgica, Hospital Central del Estado; ${ }^{3}$ Facultad de Medicina y Ciencias Biomédicas, Universidad Autónoma de Chihuahua. Chihuahua, México

\begin{abstract}
Resumen
Antecedentes: Las lesiones traumáticas de esófago tienen una prevalencia menor del 1\% y el trauma laringotraqueal representa aproximadamente 1 caso por 30,000 pacientes en el Servicio de Urgencias. Caso clínico: Paciente de sexo masculino de 33 años de edad, sin antecedentes de importancia, el cual acude a urgencias tras autolesionarse con arma blanca a nivel de cuello, se estabiliza y posteriormente ingresa a cirugía urgente para exploración de cuello y reparación de tráquea y esófago. Conclusión: Las lesiones traqueoesofágicas tienen alta mortalidad, su manejo es un desafío para el equipo quirúrgico.
\end{abstract}

Palabras clave: Lesión de tráquea y esófago. Trauma de cuello. Cirugía de trauma.

\section{Abstract}

Background: The prevalence of traumatic wounds of the esophagus is less than $1 \%$ and the incidence of laryngo-tracheal trauma is about 1 per 30,000 patients who come at emergency room, with multiple expected complications. Clinical case: A 33-year-old male patient, with no relevant medical history, hurt himself with a knife at neck level; he was stabilized in the emergency room and underwent urgent surgery for neck exploration and repair of the trachea and esophagus. Conclusion: The traumatic wounds that compromise the entire diameter of the trachea and esophagus have high mortality rates and represent a challenge for the surgeon.

Key words: Trachea and esophagus injury. Neck trauma. Trauma surgery.

Correspondencia:

*Luis B. Enríquez-Sánchez

E-mail: investigacionhcu@gmail.com
Disponible en internet: 14-01-2021 Rev Hosp Jua Mex. 2020;87(4):188-192

www.revistahospitaljuarez.com 1405-9622/@ 2020 Sociedad Médico-Quirúrgica del Hospital Juárez de México, A.C. Publicado por Permanyer. Este es un artículo open access bajo la licencia CC BY-NC-ND (http://creativecommons.org/licenses/by-nc-nd/4.0/). 


\section{Introducción}

Las lesiones traumáticas de esófago son poco frecuentes, con una prevalencia del $0.02 \%$, mayor en hombres jóvenes (entre 30 y 35 años) y menor en los extremos de la vida. Principalmente son causadas por trauma penetrante hasta en un $50 \%$, de los cuales el $35 \%$ es por proyectil de arma de fuego y el $14.9 \%$ por arma blanca ${ }^{1,2}$.

Anatómicamente el esófago abarca tres porciones: cervical, torácica y abdominal, lo que le da una irrigación característica y hace que las lesiones por mecanismos de aceleración-desaceleración sean poco frecuentes, sin embargo con un alto índice de mortalidad.

La región cervical es la porción más frecuentemente lesionada tanto por mecanismos de trauma cerrado como penetrante ${ }^{3}$. Para su estudio se describen tres zonas del cuello (Monson):

- Zona 1. De las clavículas al cartílago cricoides.

- Zona 2. Entre el cartílago cricoides y el ángulo de la mandíbula.

- Zona 3. Entre el ángulo de la mandíbula y la base del cráneo ${ }^{4}$.

Las lesiones en la zona 2 son las que mayormente requieren manejo quirúrgico. A la exploración clínica se deberán buscar signos duros (hemorragia activa, compromiso de vía aérea, hematoma pulsátil o evolutivo) y blandos (disnea, disfagia, hemoptisis), los cuales nos guiarán para valorar si es factible realizar una exploración quirúrgica o no, teniendo una sensibilidad del 100\% y una especificidad del $94.4 \%$, y una sensibilidad del $100 \%$ y especificidad del $75.5 \%$, respectivamente ${ }^{5}$.

Las venas constituyen las estructuras más comprometidas en lesiones penetrantes de cuello, pudiendo tener un manejo no quirúrgico selectivo, en comparación con las arteriales, que no ceden en su sangrado debido a la presión de las arterias ${ }^{6}$. El trauma laringotraqueal es igualmente raro, aproximadamente 1 caso por cada 30,000 pacientes que ingresan al Servicio de Urgencias. En pacientes con sospecha de lesión esofágica hay que iniciar inmediatamente antibioticoterapia, por el alto riesgo de desarrollar mediastinitis, así como una vía de nutrición alterna a la vía oral. En caso de que haya una perforación no observada, si el paciente llega dentro de las primeras 12 horas se debe intentar un cierre primario, reportándose tasas de éxito similares tanto si se realiza cierre primario en dos planos como en uno?.

Los estudios de imagen deben realizarse si las condiciones del paciente lo permiten, de lo contrario en

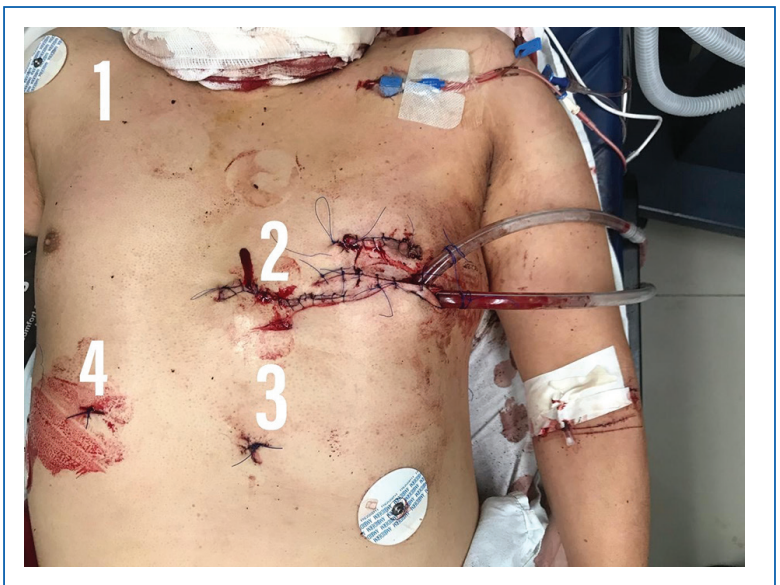

Figura 1. Paciente al ingreso. Se muestran las lesiones: 1) lesión en cuello, 2) lesiòn en tórax, 3) lesión en epigastrio y 4) lesión subcostal derecha. Ya se encuentra colocada la sonda pleural y el catéter venoso subclavio izquierdo.

caso de sospechar inestabilidad por la lesión se deberá optar por manejo quirúrgico de inmediato. El sitio de lesión asociado más común es el esófago, la lesión total de tráquea usualmente es fatal y los pacientes rara vez sobreviven ${ }^{8}$. En cuanto a la reparación, cuando hay compromiso de más del $50 \%$ de la tráquea en el adulto o más del $30 \%$ en los pacientes pediátricos no es posible hacer una anastomosis terminoterminal y se optará por un reemplazo de esta, pudiendo usar prótesis de silicona con dacrón o titanio, entre otros materiales, o un aloinjerto de aorta abdominal ${ }^{8}$.

\section{Materiales y métodos}

Hombre de 33 años de edad, el cual acude al Servicio de Urgencias del Hospital Universitario por aparente intento de suicidio con arma blanca. A su ingreso se presenta hipotenso, taquicárdico y taquipneico. Durante la exploración se evidencia lesión en región cervical anterior de cuello de $10 \mathrm{~cm}$ aproximadamente, lesión en hemitórax izquierdo a nivel del pezón e inferior a este, así mismo con heridas en epigastrio, en región subcostal derecha y en ambas muñecas (Fig. 1). Se decide manejo avanzado de la vía aérea a través de lesión traqueal, se encuentra con exposición de tráquea y esófago (Fig. 2). Se realiza tomografía simple y contrastada de cráneo, cuello, tórax y abdomen, con evidencia de neumotórax izquierdo del $40 \%$ y lesión de vena yugular interna izquierda. 


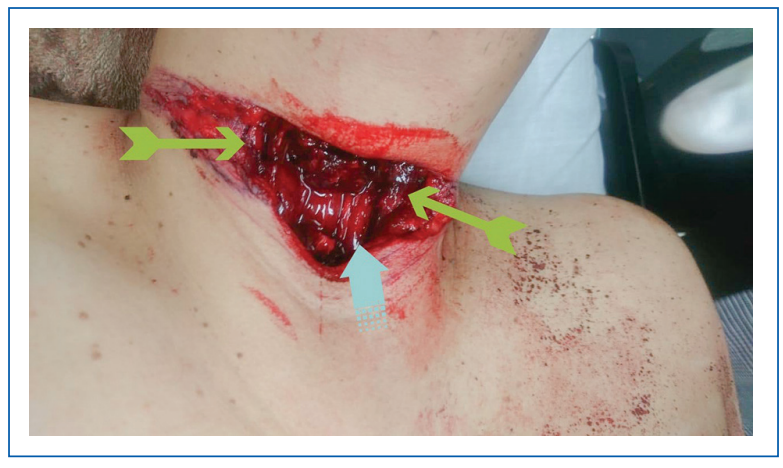

Figura 2. Lesión en cuello a nivel cervical. Flechas verdes: músculos esternocleidomastoideos bilaterales. Flecha azul: espacio prevertebral.

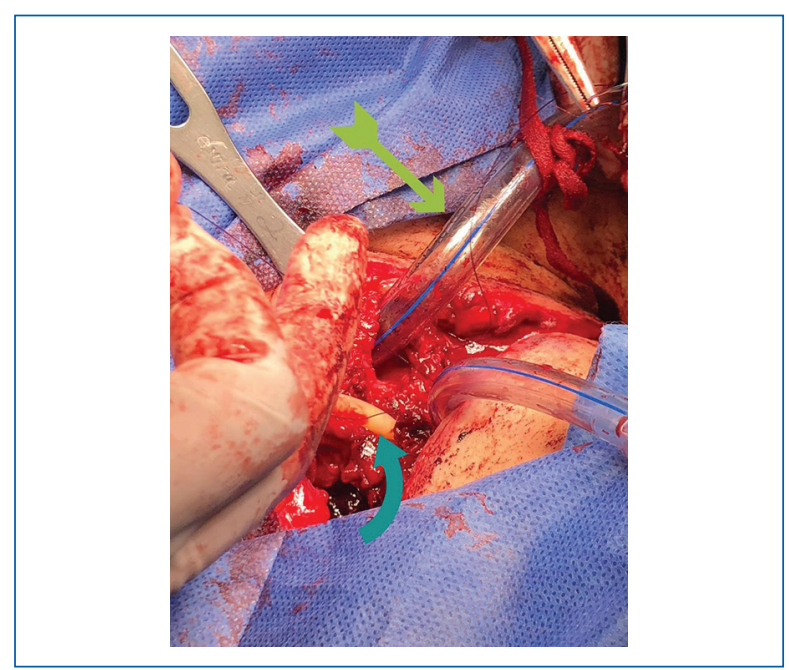

Figura 3. Flecha verde: referencia de tráquea. Flecha azul: sonda de Kehr en esófago.

Se realizó exploración quirúrgica, donde se observó lesión en tráquea y esófago en su totalidad, músculos infrahioideos, glándula tiroides y de vena yugular interna izquierda con datos de trombosis (Fig. 3); se procedió a reparar tráquea con anastomosis terminoterminal y colocación de cánula de Shiley, y a la reparación del esófago, el cual presentaba lesión total (grado $\mathrm{V}$ de acuerdo con la AAST [Asociación Americana de Cirugía de Trauma, por sus siglas en ingles]), con cierre primario y esofagostomía con colocación de sonda en T (Fig. 4), así como gastrostomía y se traslada a unidad de terapia intensiva.

Durante su internamiento se reportan cultivos positivos (hemocultivos y de secreción bronquial) a Klebsiella, Staphylococcus aureus y Acinetobacter baumannii multirresistente, por lo que se inicia

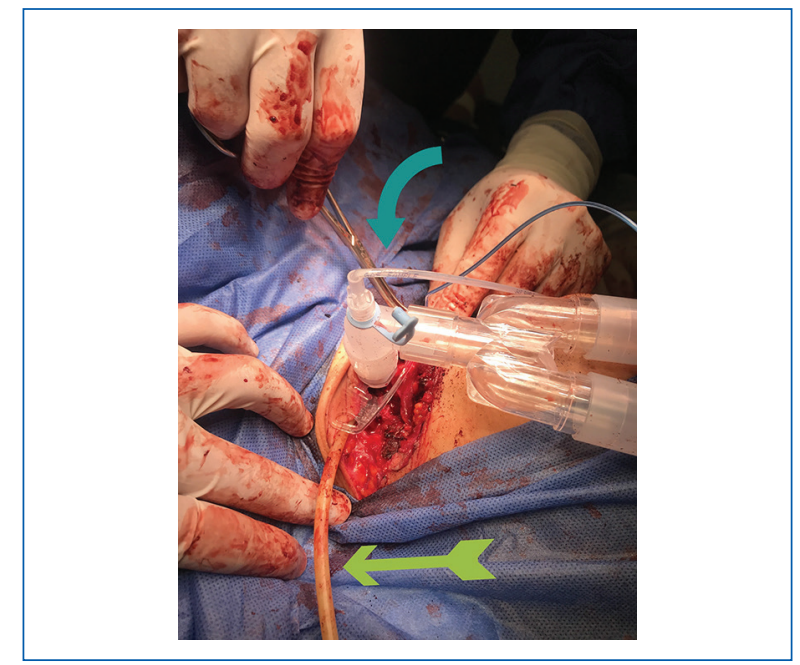

Figura 4. Flecha azul: cánula de Shiley después de la reparación de tráquea. Flecha verde: sonda de Kehr para esofagostomía, posterior a reparación esofágica.

colistimato, imipenem y linezolida, así como manejo en área aislada.

Posteriormente se realizó tomografía computarizada de control, con evidencia estenosis traqueal (Fig. 5), por lo que el paciente fue sometido, en un segundo tiempo, a una dilatación endotraqueal por el Servicio de Cirugía cardiotorácica, así como a toracotomía por hemotórax coagulado, secundario a las lesiones presentadas, ambas sin complicaciones posteriores (Fig. 6). A los 17 días postoperatorios se reportaron los cultivos negativos. A los 22 días se inicia vía oral sin problema; se evidenció fuga por traqueostomía, sospechando fístula traqueoesofágica, por lo que se realiza endoscopia, en la cual se observa solo úlcera de hipofaringe; se sospechó de alteración en la movilidad de epiglotis por el tiempo de intubación, manejado con medidas conservadoras, su evolución es favorable y se egresa.

\section{Resultados}

Paciente de sexo masculino con lesión penetrante en región anterior de cuello que presentó condiciones hemodinámicas suficientes para valoración radiológica con tomografía axial computarizada simple y contrastada con evidencia de lesión traqueoesofágica completa, por lo que fue intervenido con exploración quirúrgica urgente, donde se hace reparación primaria de esófago con esofagostomía y colocación de sonda T más gastrostomía, además de colocación de cánula de Shiley vía traqueal. El paciente es ingresado a la unidad de terapia intensiva 


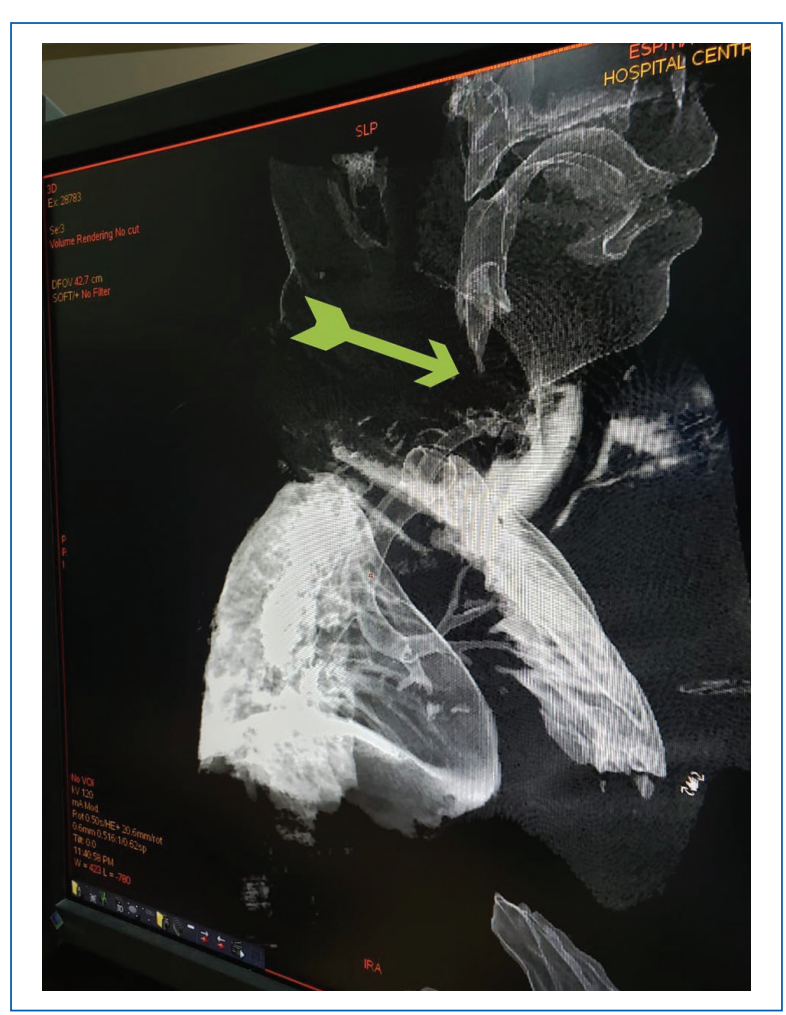

Figura 5. Reconstrucción traqueal por el Servicio de Radiología. Flecha verde: se observa estenosis traqueal.

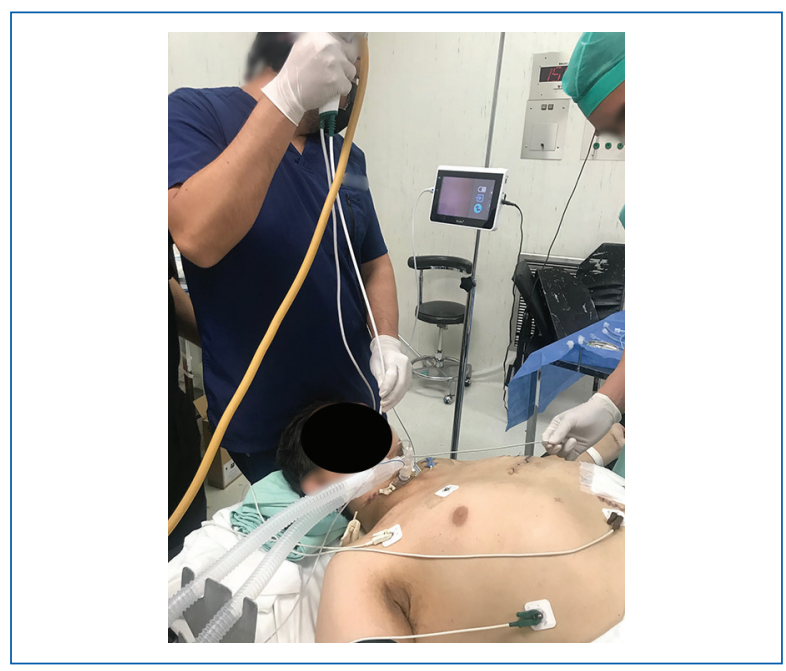

Figura 6. Realizando fibroscopia por el Servicio de Cirugía Cardiotorácica, previa dilatación traqueal.

del hospital, donde se dan medidas de soporte y manejo antibiótico por reporte de cultivos positivos; ante estenosis traqueal se realiza dilatación. Tras evolución favorable se da de alta al paciente.

\section{Discusión}

Se trata de paciente con trauma penetrante de cueIlo, con lesión total de tráquea y esófago, además de lesión de la yugular interna, a nivel de zona 2. En los reportes de caso la lesión vascular se presenta hasta en un $50 \%$, pero con predominio en la zona $1^{9}$. Durante su manejo quirúrgico se evidencia lesión grado $\mathrm{V}$ tanto de esófago como de tráquea, de acuerdo con la AAST; por el tiempo de evolución y el tipo de lesión, se decide su manejo con cierre primario. De acuerdo con las recomendaciones de la Western Trauma Association se realizó una esofagostomía con colocación de tubo en T para evitar lesiones del tejido anastomosado con la secreción salival y que aumente el riesgo de fuga ${ }^{10}$. Siempre se debe optar por la vía enteral de alimentación sobre la nutrición parenteral, por lo que se deja sonda de gastrostomía para el inicio de alimentación temprana.

Caronia, et al. (2017) reportan que la generación de fístula traqueoesofágica adquirida se da hasta en una cuarta parte de todos los casos secundaria a una afección benigna, dentro de las cuales se encuentran: ventilación mecánica prolongada (47\%), lesión (17\%), laringectomía previa (17\%) y esofagostomía (17\%). Nuestro paciente tenía alto riesgo de presentar fístula traqueoesofágica o fuga secundaria a ventilación mecánica prolongada, sin embargo no fue el caso ${ }^{11}$.

A su egreso, el paciente presenta disfonía, lo cual es una complicación esperada en intervenciones quirúrgicas de cuello anterior. Yu, et al. (2020) reportan una tasa general de disfonía del $23.1 \%{ }^{12}$.

Hubo sospecha de fístula traqueoesofágica. Se reporta que el mejor método para evidenciar las fístulas o fugas es por endoscopia. En los estudios contrastados puede evidenciarse hasta en un $70 \%$ esta complicación ${ }^{13}$. Brooks, et al. (2018) reportan que la tasa de éxito es de un 83 a un $88 \%$ con un stent autoexpandible, con una mortalidad del 7.5 al $17 \%$. Se encuentran múltiples materiales protésicos, así como injertos ${ }^{14}$.

En cuanto a la estenosis, se ha reportado una incidencia de hasta un $20 \%$ postintubación. El paciente tenía varios factores de riesgo: el tiempo que permaneció intubado, la lesión traqueal con la que llega y el posterior riesgo al estar colocando las cánulas de Shiley. La fisiopatología de la estenosis es causada por cambio histopatológicos, hemorragias en mucosa y ulceraciones, ocasionando la exposición de los anillos cartilaginosos de la tráquea y posterior fibrosis. Todos estos cambios los ocasiona un depósito anormal de 
colágeno tipo I, III y factor de crecimiento transformante beta, relacionado con la activación de miofibroblastos. Posterior a la descanulación, puede presentarse estenosis de entre cuatro y ocho semanas ${ }^{15,16}$.

La mortalidad del paciente incrementa sustancialmente ante la posibilidad de una fuga anastomótica, es por ello que es un verdadero reto para los cirujanos. Messager, et al. (2018) reportan una incidencia en los pacientes con anastomosis del 5 al $30 \%$ y esta aumenta al doble cuando su diagnóstico se realiza posterior a las 24 horas.

La disfagia presentada en lesiones de cuello es común posterior o durante su tratamiento, incluyendo otro tipo de lesiones que ocasionan parálisis de cuerdas vocales y edema supraglótico, así como las lesiones en columna asociadas.

Este tipo de casos son muy aislados y con alta mortalidad. Su manejo es un reto para todo el personal de salud y con múltiples complicaciones esperadas.

Se inició manejo como con todo paciente en estado crítico, asegurando la vía aérea. La rápida intervención del equipo quirúrgico, así como el manejo en conjunto con los servicios de cirugía cardiotorácica, otorrinolaringología, anestesiología, terapia intensiva, psiquiatría, infectología y psicología. El paciente se egresa sin cánula de traqueostomía y tolerando vía oral sin problema.

\section{Conclusión}

El manejo del paciente con trauma penetrante de tórax sigue siendo un reto para los servicios médico quirúrgi$\cos$, ya que requiere de una rápida actuación diagnóstica y clínica para discriminación de lesiones vitales importantes para dirigir el tratamiento más indicado.

\section{Financiamiento}

El desarrollo del presente caso no ha recibido ayudas específicas provenientes de agencias del sector público, sector comercial o entidades sin ánimo de lucro.

\section{Conflicto de intereses}

Los autores declaran no tener conflicto de intereses alguno.

\section{Responsabilidades éticas}

Protección de personas y animales. Los autores declaran que para esta investigación no se han realizado experimentos en seres humanos ni en animales.

Confidencialidad de los datos. Los autores declaran que han seguido los protocolos de su centro de trabajo sobre la publicación de datos de pacientes.

Derecho a la privacidad y consentimiento informado. Los autores han obtenido el consentimiento informado de los pacientes y/o sujetos referidos en el artículo. Este documento obra en poder del autor de correspondencia.

\section{Bibliografía}

1. Aiolfi A, Inaba K, Recinos G. Non-iatrogenic esophageal injury: a retrospective analysis from the National Trauma Data Bank. World J Emerg Surg.2017;12:19

2. Olding J, Olding C, Fan K. Penetrating head \& neck trauma - Epidemiology and injury characteristics in terror-related violence, interpersonal violence and deliberate self-harm at a level 1 trauma centre. Surgeon. 2019;17(3):133-8

3. Petrone $P$, Kassimi K, Jiménez-Gómez M, Betancourt A, Axelrad A, Marini CP. Management of esophageal injuries secondary to trauma. Injury. 2017;48(8):1735-42

4. Alao T. Neck Trauma [Internet]. StatPearls [actualizado: 28/02/2019; citado: 29/05/2019]. Disponible en: https://knowledge.statpearls.com/chapter/0/25616?utm_source=pubmed\#References

5. Tessler R, Nguyen H, Newton C. Pediatric penetrating neck trauma: hard signs of injury and selective neck exploration. J Trauma Acute Care Surg. 2017;82(6):989-94.

6. Madsen A, Bruce J, Oosthuizen G. The selective non-operative management of penetrating cervical venous trauma is safe and effective. World J Surg. 2018;42(10):3202-9.

7. Nowicki JL, Stew B, Ooi E. Penetrating neck injuries: a guide to evaluation and management. Ann R Coll Surg Engl. 2018;100:6-11.

8. Etienne H, Fabre D, Gomez A.Tracheal replacement. Eur Respir J. 2018;51(2):1702211.

9. Biffl WL, Moore EE, Feliciano DV, Albrecht RA, Croce M, Karmy-Jones R, et al. Western Trauma Association critical decisions in trauma: diagnosis and management of esophageal injuries. J Trauma Acute Care Surg. 2015;79(6):1089-95

10. Caronia FP, Reginelli A, Santini M, Alfano R, Trovato S, Arrigo E, et al. Trans-tracheostomy repair of tracheo-esophageal fistula under endoscopic view in a 75-year-old woman. J Thorac Dis. 2017;9(3):E176-E179.

11. Yu S, Chen Z, Yan N, Hou T, He S. Incidence and factors predictive of dysphagia and dysphonia after anterior operation with multilevel cervical spondylotic myelopathy. Clin Spine Surg. 2017;30(9):E1274-E1278.

12. Santosham R. Management of acquired benign tracheoesophageal fistulae. Thorac Surg Clin. 2018;28:385-92.

13. Brooks U, Douglas JM, Harald CO. Bioprosthetics and repair of complex aerodigestive defects. Ann Cardiothorac Surg. 2018;7(2):284-92.

14. Cooper JD. Tracheal injuries complicating prolonged intubation and tracheostomy. Thorac Surg Clin. 2018;28:139-44.

15. Enyuan Q, Mingpeng X, Luoman G. Erythromycin combined with corticosteroid reduced inflammation and modified trauma-induced inflammation and modified trauma-induced tracheal stenosis in a rabbit model. Ther Adv Respir Dis. 2018:12:1-13.

16. Persson S, Rouvelas I, Lundell L. Outcomes following the main treatment options in patients with leaking esophagus: a systematic literature review. Dis Esophagus. 2017;30:1-10. 